\title{
Overspecification in action-oriented discourse: task importance affects the production of overspecifications and overspecifications increase identification efficiency in perception
}

Anja Arts ${ }^{1}$, Alfons Maes ${ }^{1}$, Leo Noordman ${ }^{1}$ and Carel Jansen ${ }^{2}$

${ }^{1}$ Faculty of Humanities, Tilburg University, the Netherlands

${ }^{2}$ Faculty of Arts, Radboud University, the Netherlands

https://doi.org/10.36505/ExLing-2008/02/0008/000067

\begin{abstract}
This paper discusses the effect of overspecified reference as a communicative strategy in action-oriented discourse. In a perception and a production experiment, based on identical instructive environments, participants were asked to identify an object after reading a minimally specified or overspecified expression, or to produce a referential expression in a low-importance or a high-importance context. Overspecification shows to be a pervasive instructive production strategy, which mostly affects the addressee's identification task in a positive way.
\end{abstract}

Key words: overspecification, instruction, coherence, reference

\section{Introduction}

Cognitively oriented research of discourse reference aims to explain and predict the form and specification level of referential expressions in discourse. It centers on the discourse structural factors that affect the cognitive status - and consequently also the specification level - of discourse referents (Ariel, 1991; Gundel et al., 1993).

The mechanism of referential coherence is complicated considerably in less 'autonomous' conditions of language use, in particular when language is more closely integrated with perception, action and participants' interaction. We address the issue of overspecification (i.e. providing more referential information than is necessary for unambiguous identification) as a referential strategy in task and action oriented discourse.

Instructors in a non-feedback situation tend to display a considerable degree of referential overspecification in explaining procedures and actions to be executed (Maes et al., 2004). Clark and Wilkes-Gibbs (1986) offer an elegant and plausible explanation of this overspecification strategy in formulating the principle of distant responsibility ${ }^{1}$. This producer-oriented explanation, however, leaves at least two questions unanswered. First, how does this producer's strategy relate to the referential needs of the addressee? Is it to be considered a useless but harmless addition, or is it beneficial and really required for the current purposes of the exchange? Second, what are

ExLing 2008: Proceedings of 2nd Tutorial and Research Workshop on Experimental Linguistics, 25-27 August 2008, Athens, Greece 
the situational and interaction-oriented parameters that determine the nature and the extent of the overspecification?

\section{Method}

The research questions were addressed in two experiments, in an identical instructive context (for full details see Arts, 2004). For this purpose, computer screens were designed, consisting of a panel with four objects. The objects could differ in location, shape, color and size. Figure 1 provides an example computer screen.

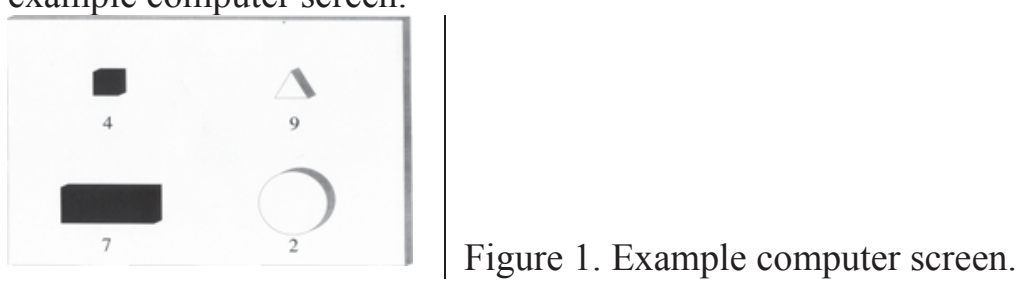

\section{Material}

Perception experiment - The design of the computer screens accommodated the use of seven minimally specified expressions (Table 1). These expressions contained just the necessary information for identification of the referent, but not more information. Every minimally specified expression could be expanded using one or more of the remaining types of information units still available for reference. This led to a total of twenty possible overspecified expressions.

Table 1. Minimally specified expressions and information units.

$$
\text { ident. vert. horiz. }
$$

nr. shape size color axis axis example

\begin{tabular}{|c|c|c|c|c|c|}
\hline $\begin{array}{ll}1 & \sqrt{ }\end{array}$ & & & & & the round button \\
\hline 2 & $\sqrt{ }$ & $\sqrt{ }$ & & & the large white button \\
\hline 3 & $\sqrt{ }$ & & $\sqrt{ }$ & & the large button at the top \\
\hline 4 & $\sqrt{ }$ & & & $\sqrt{ }$ & the large button on the left \\
\hline 5 & & $\sqrt{ }$ & $\sqrt{ }$ & & the white button at the top \\
\hline 6 & & $\sqrt{ }$ & & $\sqrt{ }$ & the white button on the left \\
\hline 7 & & & $\sqrt{ }$ & $\sqrt{ }$ & the button at the top left \\
\hline
\end{tabular}

Production experiment - For the production experiment the computer screens were adapted in two ways. Firstly, the numbers that identified the objects were removed, and secondly, one of the objects was marked with an X. 


\section{Procedure}

Perception experiment - 56 students were presented with the computer screens and asked to identify one of the four objects on the basis of one of the twenty-seven possible types of referential expressions (seven minimally specified and twenty overspecified).

Production experiment - 25 students in the low-importance condition and 27 students in the high-importance condition were presented with the computer screens and asked to refer to one of the four objects (highimportance: "indicate which button the surgeon has to push next"; lowimportance: "type in which object is marked on the screen").

\section{Results}

Perception experiment - The identification time was measured in milliseconds. The data were analyzed using one-way analyses of variance with level of specification (minimally specified, overspecified) as withinsubjects factor. The results showed that an expansion of a minimally specified expression with a reference to the vertical axis or a reference to both axes led to shorter identification times. An expansion with a sole reference to the horizontal axis did not affect the identification time. Furthermore, an expansion of a minimally specified expression with additional size, color, or shape information only led to shorter identification times if the expansion rendered the referential expression exhaustive in attribute-based information: when shape as well as color as well as size were mentioned.

Production experiment - The data were analyzed using a t-test for independent samples. In analyzing the referential expressions that were produced, the focus was on the total number of information units used (Figure 2) and on the type of information units used (Figure 3 and Figure 4) in building the referential expression.

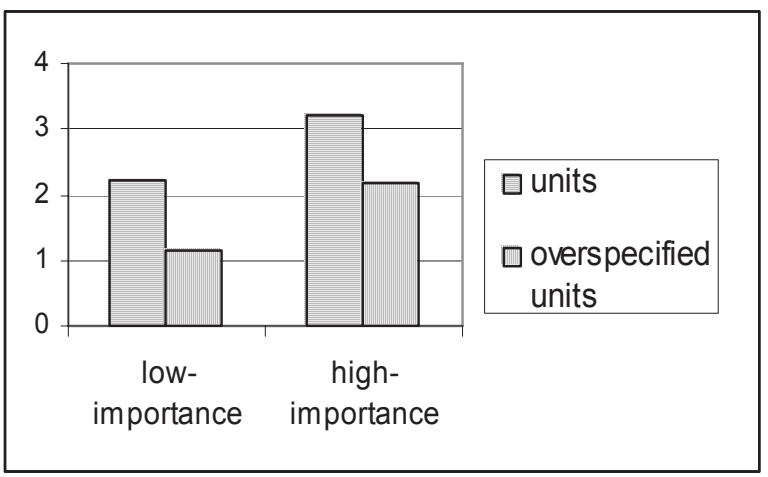

Figure 2. Total units and overspecified units per description. 


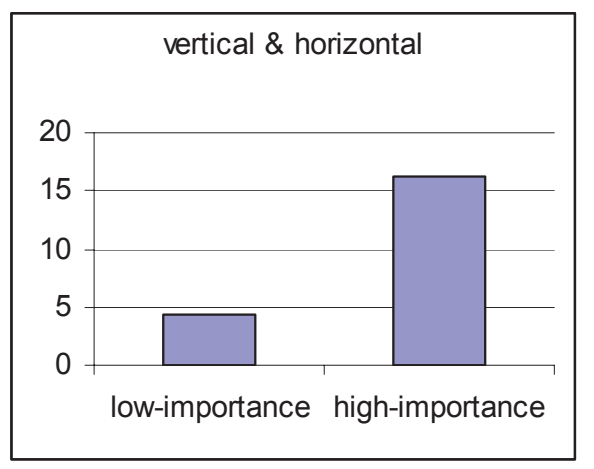

Figure 3. Objects (max. 30) described Figure 4. Objects (max. 30) described by referring to both location axes.

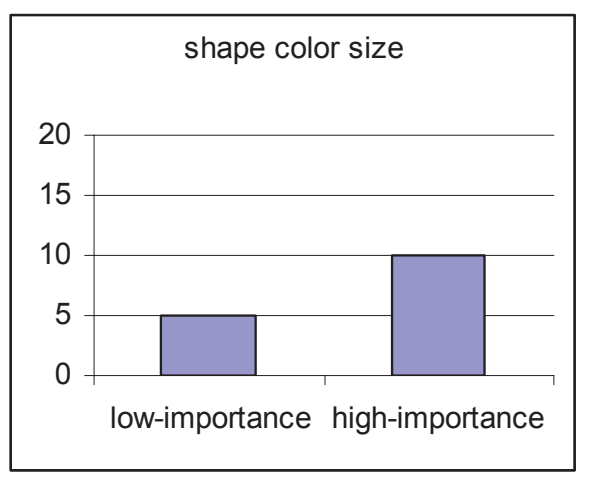
by referring to shape, color and size.

\section{Discussion}

The processing task (perception) is affected by the use of minimally specified versus overspecified referential expressions, and referential production is affected by the producer's task orientation. Though overspecification undoubtedly increases reading time, it does not decrease the identification efficiency. These results indicate that the producer's task orientation and distant responsibility, rather than possibly the complexity of an instructive task or the assumed experience level of an addressee, are responsible for the overspecification strategy.

\section{Notes}

${ }^{1}$ The principle of distant responsibility refers to the fact that, in a non-feedback situation, the language recipient can not ask for clarification. This may cause the language producer to be highly informative (Clark and Wilkes-Gibbs, 1986).

\section{References}

Ariel, M. 1991. The function of accessibility in a theory of grammar. Journal of Pragmatics 16, 443-463.

Arts, A. 2004. Overspecification in instructive texts. Doctoral dissertation, Tilburg University, the Netherlands.

Clark, H. and Wilkes-Gibbs, D. 1986. Referring as a collaborative process. Cognition 22, 1-39.

Gundel, J., Hedberg, N. and Zacharski, R. 1993. Cognitive status and the form of referring expressions in discourse. Language 69, 274-307.

Maes, A., Arts, A. and Noordman, L. 2004. Reference management in instructive discourse. Discourse Processes 37, 117-144. 